\title{
Tangence
}

\section{La perception de l'Autre}

Jalons pour une critique littéraire interculturelle

\section{Hans-Jürgen Lüsebrink}

Numéro 51, mai 1996

Paradigmes critiques

URI : https://id.erudit.org/iderudit/025904ar

DOI : https://doi.org/10.7202/025904ar

Aller au sommaire du numéro

Éditeur(s)

Tangence

ISSN

0226-9554 (imprimé)

1710-0305 (numérique)

Découvrir la revue

Citer cet article

Lüsebrink, H.-J. (1996). La perception de l'Autre : jalons pour une critique

littéraire interculturelle. Tangence, (51), 51-66. https://doi.org/10.7202/025904ar d'utilisation que vous pouvez consulter en ligne.

https://apropos.erudit.org/fr/usagers/politique-dutilisation/ 


\title{
La perception de l'Autre. Jalons pour une critique littéraire interculturelle*
}

\author{
Hans-Jürgen Lüsebrink
}

\section{1-Dimensions anthropologiques /réfractions historiques}

Le rapport dialectique entre identité collective et perception de l'Autre représente une des composantes essentielles du discours littéraire et culturel, et par conséquent un des défis majeurs de l'ensemble des paradigmes critiques qui s'y rattachent. Placée dans la perspective d'une archéologie de la communication littéraire et culturelle, cette constellation fondatrice a été, en effet, à la fois génératrice de profils sémantiques de personnages littéraires, picturaux et cinématographiques, de rôles culturels ("l'étranger", "l'exote", "l'indigène"), de schémas narratifs, de réseaux de personnages et, enfin et surtout, de genres spécifiques: le récit de voyage, en premier lieu; la description et le récit ethnographiques; le dialogue philosophique avec l'Autre, fortement pratiqué au xviI ${ }^{\mathrm{e}}$ siècle et pour lequel les Dialogues avec un Sauvage (1704) de La Hontan constituèrent un modèle exemplaire; et, puis, des pans entiers de genres romanesques, de la peinture, de la publicité et du film formant autant de sous-genres focalisés sur la perception de l'Autre, comme le roman colonial et le roman d'aventures, la publicité touristique, la peinture coloniale, le film exotique et le manuel de voyage.

Derrière l'historicité des genres et formes littéraires et culturels particuliers, et souvent spécifiques pour une époque particulière (tels le dialogue philosophique pour le $\mathrm{xvII}^{\mathrm{e}}$ et la peinture coloniale pour le $\mathrm{XIX}^{\mathrm{e}}$ et les premières décennies du $\mathrm{xx}^{\mathrm{e}}$ siècle), se dessine la permanence de dispositifs psychologiques et anthropologiques. Ceux-ci régissent, avec des accentuations diverses, la perception de l'Autre et sa relation avec des formes d'identité collective, dans toutes les sociétés, au moins celles de mouvance occidentale. On pourrait en distinguer essentiellement trois :

* Le présent article a bénéficié des travaux et débats d'un séminaire dirigé par l'auteur à l'Université de Saarbrücken (Allemagne) en 1995-96 et d'une conférence (suivie d'un débat) organisée en commun avec Maguèye Kassé, à l'Université Cheikh Anta Diop de Dakar en mars 1996. Je remercie M. Kassé et les participants aux discussions de leurs suggestions et de leurs critiques. 
- En premier lieu, la fascination pour l'Autre, en particulier pour l'esthétique et les formes et conditions de vie différentes. On la trouve ancrée, de manière quasi archétypale, dans le journal de bord de Christophe Colomb qui fait traverser son discours sur les habitants des Isles Caraïbes par des rêférences bibliques au paradis et reste ébloui, surtout dans les notices relatives aux premières rencontres avec les Indiens, par leur beauté physique ${ }^{1}$. Depuis le $\mathrm{xvI}^{\mathrm{e}}$ siècle, et la découverte du Nouveau-Monde, les mondes lointains et leurs habitants, mais aussi parfois des civilisations plus proches sont ainsi devenues à la fois des objets de curiositẻ et de fascination. Celles-ci se prolongent dans des formes de discours qui englobent aussi bien la littérature écrite proprement dite qu'une multiplicité de genres et de pratiques culturels: tels l'exotisme, le primitivisme, l'anglomanie française du $\mathrm{XvIII}^{\mathrm{e}}$ siècle, la germanophilie française impliquant un ensemble de représentations de l'Allemagne et des Allemands en France depuis le début du $\mathrm{xIx}^{\mathrm{e}}$ siècle, et la francophilie d'intellectuels américains et allemands des $\mathrm{xxx}^{\mathrm{e}}$ et $\mathrm{xx}^{\mathrm{e}}$ siècles. Dans le roman Deutscbland (1929) de René Trintzius, par exemple, les deux personnages féminins, amoureux d'un jeune Français stagiaire à Berlin, ont en commun une image tout à fait idéalisée de la France, "cette France idéale qui [...] obsède l'Allemagne d'un amour à la fois transi, irrité, irritant et malheureux" "2, et qui est considérée comme radicalement opposée à la culture et au style de vie de l'Allemagne prussienne. Ce dispositif psychologique et anthropologique renferme la projection de désirs, résultants de manques perçus dans sa propre culture, sur des cultures étrangères, et souvent très lointaines. S'y dessine ainsi une étroite interdépendance entre auto-image et bétéro-image, selon la terminologie de l'imagologie comparatiste ${ }^{3}$.

1 Voir pour une approche interdisciplinaire, en l'occurrence littéraire, historique et anthropologique: Stephen Greenblatt, Marvellous Possessions. The Wonder of the New World, Chicago, University of Chicago Press, 1991.

2 René Trintzius, Deutschland. Roman (1929), Rééd. Paris, Editions Phébus, 1995 , p. 82.

3 Voir notamment Manfred S. Fischer, "Komparatistische Imagologie. Für eine interdisziplinäre Erforschung national-imagotyper Systeme», Zeitschrift für Sozialpsychologie, $\mathrm{n}^{\circ} 10,1979$, p. 30-44; Manfred S. Fischer, Nationale Images als Gegenstand vergleichender Literatungeschichte. Untersuchungen zur Entstebung der Imagologie, Bonn, 1981 ; le numéro thématique de la revue Komparatistische Hefte (Université de Bayreuth), 2, 1980; Hugo Dyserinck, -Zum Problem der "images" und "mirages" und ihrer Untersuchung im 
- En second lieu, la perception de l'Autre peut être étroitement liée, sur le plan anthropologique, à des processsus de négation et d'exclusion qui sont générateurs de territoires d'identités. Impliquant une tendance vers l'ontologisation des différences, ces processus que l'on trouve tracés dès l'Antiquité gréco-romaine, dans l'œuvre de Tacite par exemple ${ }^{4}$, sont constitutifs non seulement de différences, généralement culturelles et linguistiques, mais aussi de clivages culturels. Sigmund Freud et, dans son sillage, l'ethnopsychologue Mario Erdheim ${ }^{5}$, ont démontré l'interrelation anthropologique entre des formes, mêmes radicales, d'exclusion, comme la xénophobie, et des formes d'attirance et de fascination, comme l'exotisme, puisqu'elles ont en commun de semblables stratégies d'évitement psychologiques ("Vermeidungsstrategien") détournées de toute tentative sérieuse de compréhension et de connaissance de l'Autre. C'est dans ce sens précis que la perception de l'Autre, aussi bien son idéalisation exotisante que son rejet et son exclusion, s'avèrent, comme le postule Julia Kristeva ${ }^{6}$, être la face cachée de notre identité, liée aux désirs d'évasion ou de refoulement. Le poète expressionniste allemand Georg Heym les exprima en souhaitant en 1910, vu que tout était extrêmement ennuyeux en Europe, une "Révolution", une "Guerre" ou encore une "traversée de l'Afrique"?. Franz Kafka a tracé, dans son roman Die Strafkolonie, à partir de

Rahmen der vergleichenden Literaturwissenschaft ", Arcadia, n ${ }^{\circ} 1,1966, \mathrm{p}$. 107-120; et sur l'image de l'Allemagne dans la littérature française en particulier l'étude de Wolfgang Leiner, Das Deutschlandbild in der französischen Literatur, Darmstadt, Wissenschaftliche Buchgesellschaft, $2^{\mathrm{e}}$ éd. 1991, qui est paradigmatique pour une approche 'imagologique' de la représentation de l'Autre dans la littérature, en particulier dans les études et départements de littérature comparée.

4 Michael Werner, "La Germanie de Tacite et l'originalité allemande ", Le débat, dossier thématique "Mémoires comparées", no 78, janvier-février 1994, p. 4261.

5 Sigmund Freud, "Das Unheimliche", S. Freud, Studienausgabe, t. IV : Psychologische Scbriften. Ed. par Alexander Mitscherlich, Frankfurt/M., Fischer, $7^{\mathrm{e}}$ éd. 1970, p. 241-275; Mario Erdheim, "Zur Ethnopsychoanalyse von Exotismus und Xenophobie", Exotische Welten - Europäische Phantasien, Ed. Hermann Pollig, Stuttgart, Edition Crantz, 1987, p. 48-54.

6 Julia Kristeva, Etrangers à nous-mêmes, Paris, Seuil, 1988

7 Georg Heym, Dichtungen und Scbriften. T. 3: Tagebücher, Träume, Briefe. München, 1960 , p. 128, 138. Voir sur ce point aussi Thomas Schwarz, "Die Tropen bin ich!" Der exotistische Diskurs der Jahrhundertwende ", Kulturrevolution. Zeitschrift für angewandte Diskurstbeorie, $\mathrm{n}^{\circ}$ thématique "Tropische Tropen - Exotismus", nos 32-33, 1995, p. 11-21. 
54

documents contemporains sur les camps punitifs coloniaux, une des fictionalisations littéraires les plus saisissantes de l'exclusion xénophobe; mais en même temps, il a manifesté, dans son récit Wunsch, Indianer zu werden ("Le désir de devenir Indien", 1915), le désir d'incorporer l'Autre à la fois psychiquement et physiquement en devenant un transfuge culturel à part entière ${ }^{8}$.

- En troisième lieu, la connaissance de l'Autre est ancrée, dans toutes les sociétés humaines, dans un dêsir à la fois de curiosité et d'information, mais aussi dans une volonté soit de maîtrise de situations interculturelles potentiellement conflictuelles, soit de domination. Des disciplines comme l'ethnologie et l'anthropologie modernes, une grande partie de la littérature coloniale et, enfin, des formes de mise en scène à première vue essentiellement esthétiques de l'Autre - comme les salons de peinture coloniale ou les expositions coloniales $^{9}$ - sont ainsi profondément marquées par ce double dispositif.

L'expansion coloniale, depuis la fin $\mathrm{du}_{\mathrm{xv}}^{\mathrm{e}}$ siècle, l'avènement du nationalisme, et les formes discursives générées par ces deux mutations culturelles et politiques fondamentales des temps modernes qui ont également eu un impact considérable sur la production littéraire, ont profondément modifié l'articulation spécifiquement historique de ces dispositifs anthropologiques. Ils ont d'abord ethnisé, surtout à travers la pensée nationaliste et l'idéologie impérialiste nées depuis le début du $\mathrm{XIX}^{\mathrm{e}}$ siècle, les formes d'exclusion et de négation observables dans toutes les cultures, de manière plus ou moins aiguë, en les ancrant dans des modèles de hiérarchisation des races et des ethnies. Si la pensée nationaliste de la Révolution française était encore fondée sur des dichotomies politiques ("nation" opposée à "aristocratie", par exemple), celle du nationalisme allemand et, dans sa prolongation épisté-

8 Franz Kafka, "Wunsch. Indianer zu werden", F. Kafka, Sämtliche Erzäblungen. Ed. par Paul Raabe. Frankfurt/M., Fischer, 1975. Voir aussi sur ce sujet Michaela Holdenrieth, "Das kannibalische Dreieck, Ikonen des Fremden", Kultur-revolution. Zeitschrift für angewandte Kulturtheorie, $\mathrm{n}^{\circ}$ thématique "Tropische Tropen - Exotismus", nos 32-33, 1995, p. 11-21; sur Kafka, p. 12.

9 Voir sur ce point Robert Debusmann et János Riesz (éd.), Kolonialausstellungen - Begegnungen mit Afrika, Frankfurt/M., IKO-Verlag für Interkulturelle Kommunikation, 1995, notamment dans cette perspective la contribution de J. Riesz: "Kolonialwaren" - Die großen Kolonialausstellungen als "exotische" Warenlager und Instrumente kolonialer Propaganda ", p. 159-178. 
mologique, celle de l'impérialisme colonial de la fin du $\mathrm{xIX}^{\mathrm{e}}$ siècle, furent marquées par l'opposition des qualités raciales qui restructura profondément à la fois la représentation de l'identité collective et les modes de perception de l'Autre. L'écrivain Ernst Moritz Arndt, un des porte-paroles majeurs du nationalisme allemand du XIX ${ }^{e}$ siècle, opposa ainsi "das Eigene" et "Das Fremde", l'essence même d'une nation et tout ce qui lui est étranger. Le " caractère allemand" ("deutsche Art") aurait été effacé par des influences étrangères, surtout françaises, qu'il s'agirait à tout prix de refouler et de réprimer - telle est la thèse fondamentale de Arndt, présente tant dans ses poèmes que dans ses écrits pamphlétaires qui débouchent sur une métaphorique de l'empoisonnement et de l'anéantissement: "Jusque dans son noyau même l'Allemand était empoisonné par l'Autre, l'étranger", écrit-il ainsi dans Über Volkshaß und das Erlernen einer fremden Sprache de 1813, en visant en particulier l'influence culturelle française, notamment littéraire et linguistique, en Allemagne; "son caractère mâle et sérieux s'était transformé en manières affectées, la haute vérité en flagornerie, la raison droite en pure sottise "10. L'ethnologue américain James Clifford a dégagé le même type de dichotomisation dans le discours colonial en relevant la forte propulsion donnée par celui-ci à "essentialiser" l'Autre, à le figer dans une différence radicale et généralement perçue et valorisée à travers une conception évolutive et téléologique des civilisations humaines ${ }^{11}$.

La hiérarchisation ethnique et raciale, promue par la pensée nationaliste du $\mathrm{XIX}^{\mathrm{e}}$ siècle et atteignant ses paroxysmes à l'époque

10 Ernst Moritz Arndt, Über Volksbaßs und den Gebraucb einer fremden Sprache, Leipzig, 1813, p. 15: "Bis in den innersten Kern vergiftet war das Teutsche von dem Fremden, die ernste Männlichkeit zu Ziererei, die hohe Wahrheit zu Schmeichelei $[\ldots]$.

11 James Clifford, "On Orientalism", The Predicament of Culture: TwentiethCentury Etbnography, Literature and Art, Cambridge, MA./London, Harvard University Press,1988, p. 255-276; ici p. 258: "If Orientalism, as Said describes it, has a structure, this resides in its tendency to dicbotomize the human continuum into we-they contrasts and to essentialize the resultant "other"to speak of the oriental mind, for example, or even to generalize about " Islam" or the "Arabs' ". Voir aussi sur ce sujet Mick Gidley, "Representing Others : an Introduction", Mick Gidley (éd.), Representing Otbers. White Views of Indigenous Peoples, Exeter, University of Exeter Press, 1992, rééd. 1994 ("Exeter Studies in American and Commonwealth Art Studies", n ${ }^{\circ} 4$ ), p. 1-13; ici p. 3. 
56

de l'impérialisme colonial et du nationalsocialisme en Allemagne, accentua ainsi la perception dichotomique de l'Autre, vue comme fondamentalement opposée à l'identité collective de soi-même et de la propre nation. Si des identités collectives stéréotypées ont toujours existé, également dans la littérature - des "Germains" de Tacite jusqu'aux nobles sauvages idéalisés par Jean-Jacques Rousseau et Chateaubriand -, celles-ci reçurent désormais un ancrage à la fois interdiscursif, politique et ethnique: interdiscursif, parce qu'elles se trouvèrent diffusées à travers une multiplicité de genres et de textes extrêmement divers, allant du vaste éventail de formes littéraires jusqu'aux discours anthropologiques et ethnographiques, mais englobant aussi la politique culturelle, les mises en scène des expositions coloniales, l'univers pictural et cinématographique, et en particulier la publicité; politique, parce qu'elles répondaient à des objectifs de domination politique régissant, de manières extrêmement diverses, en partant de l'influence directe jusqu'aux formes de contre-discours les plus radicales, la perception des rapports interculturels dans l'ensemble des discours sociaux (dont la littérature); et, enfin, etbnique, parce que les caractéristiques ethniques, pensées généralement dans le cadre des limites d'une "nation" et d'un "peuple", se donnèrent à lire comme a-historiques et essentialistes, cimentant la cohésion nationale à travers l'imaginaire d'un ensemble de traits de caractère culturels, moraux, voire physiques communs à tous ses membres. Immense machine de reproduction, mais aussi de réfraction et de subversion de modes de perception stéréotypés de l'Autre, la littérature moderne et contemporaine contribua, dans une large mesure, à ancrer ces registres dans les mentalités collectives: à travers entre autres des portraits de personnages; des schémas narratifs stéréotypés; ou encore des paysages schématisés ${ }^{12}$ destinés à renvoyer de manière métonymique aux traits de caractère ethniques de ses habitants - tel ce "grand décor africain" décrit par Pierre Loti sur les premières pages de son Roman d'un spahi (1881). Celui-ci est, à l'image du profil ethnique colonial reproduit par Loti, caractérisé par les "silhouettes

12 Voir, pour ce phénomène de la stéréotypisation nationale des paysages littéraires, l'étude de Fritz Nies, "Ein Schlüssel zum französischen Deutsch-landbild : deutsche Landschaft in französischer Literatur des 19. und 20. Jahrhunderts", Michel Grunewald/ Jochen Schlobach (éds.), Médiations/ Vermittlungen. Aspects des relations franco-allemandes du xvfe à nos jours, Frankfurt/M., Lang, 1992, t. 2, p. 247-260 
pointues des cases yolofes, massées par centaines", d'une "mer agitée" et de la "ligne éternelle des brisants d'Afrique"; d'un "soleil jaune, près de disparaître, éclairant encore d'une lueur terne le désert à perte de vue, le sable sans fin "; "une caravane lointaine de Maures, des nuées d'oiseaux de proie planant dans l'air"; et, enfin, un "cimetière", celui en l'occurrence du village de Sorr près de Saint-Louis-du-Sénégal, où le protoganiste Jean Peyral avait déjà "conduit quelques-uns de ses camarades, montagnards comme lui, morts de la fièvre, sous ce climat maudit"13.

\section{II - Défis méthodologiques - le cas de l'“exotisme ” littéraire}

L'exotisme constitue, depuis le $\mathrm{XvI}^{\mathrm{e}}$ siècle, un des paradigmes de perception dominants de l'Autre, en particulier à l'égard des sociétés et cultures lointaines, en dehors de l'Europe. Discours de représentation et d'appropriation des cultures différentes en Occident, le terme d'"exotisme " apparut dans son acception moderne au milieu du $\mathrm{XvI}^{\mathrm{e}}$ siècle, dans le sillage de l'expansion européenne outre-mer. Sa première occurrence, signalée par le dictionnaire étymologique de Bloch et Wartburg, date vraisemblablement du Quart Livre de François Rabelais où il est question de "diverses tapisseries, divers animaux, poissons, oiseaux et autres marchandises exotiques et pérégrines qui étaient en l'allée du môle et par les halles du port"14. Dérivé du mot grec "exótikos" qui signifie "étranger" ou "différent ", le terme "exotique"se réfère depuis le $x v^{e}$ siècle à tout un ensemble d'images de l'Autre, cristallisées tant dans des représentations visuelles que dans des récits et des termes y relatifs qui constituent un vaste champ lexical et sémantique: "mystérieux", "bizarre", "curieux", "enchantant", "indigène", "naturel" et "paradisiaque" en forment les éléments lexicaux les plus fréquents; tandis que, sur le plan des objets référentiels attachés à l'imaginaire de l'exotisme, en particulier dans la littérature, prédominent la forêt tropicale, le soleil et une représentation du corps humain liée aux concepts de

13 Pierre Loti, Le roman d'un spahi (1881), rééd. Paris, Calmann-Lévy/Le Livre de poche, 1987, p. 16.

14 O. Bloch et W. von Wartburg, Dictionnaire êtymologique de la langue française, cité d'après Vincenette Maigne, "Exotisme: évolution en diachronie du mot et de son champ sémantique", Exotisme et création. Actes du colloque international de Lyon, Lyon, 1985, p. 9-16. 
58

"nature", de "sensualité " et de "liberté sexuelle". Générateur de représentations visuelles et de récits multiples d'une complexité textuelle très inégale, le paradigme discursif de l'exotisme a certes trouvé son expression la plus élémentaire, et la plus influente dans les sociétés occidentales contemporaines, dans l'annonce publicitaire de voyages: comme celle, par exemple, d'un club de vacances touristique allemand nommé "Robinson Club" qui présente son "Club Baobab" à Diani Beach au Kenya à travers un texte qui reproduit, de manière très stéréotypée, un mythe exotique du continent africain : "La fascination de l'Afrique, du continent mystérieux et originel. La fascination de la vie simple, tout près de plages infinies et au milieu d'une forêt tropicale naturelle, longeant la côte, et pourvue d'une étonnante variété de plantes et d'animaux " 15

Une critique littéraire interculturelle de ce phénomène de l'exotisme peut être fondée sur trois approches méthodologiques systématiquement imbriquées: l'analyse sémiologique des formes de représentation qui les constituent; l'analyse sociocritique de ses ancrages idéologiques et sociaux; et l'analyse interdiscursive des réseaux de discours dans lesquels un texte donné, par exemple un ouvrage littéraire "exotique" par excellence" comme le roman Paul et Virginie (1788) de Bernardin de Saint-Pierre, s'intègre et prend sens.

L'analyse sémiologique, telle qu'elle a été, par exemple, développée par Roland Barthes et Philippe Hamon en France, et les travaux plus récents de Erika Fischer-Lichte en Allemagne ${ }^{16}$, s'attache à l'inventaire à la fois descriptif et fonctionnel des éléments constitutifs de la perception de l'Autre, à travers un paradigme comme l'"exotisme" par exemple : à savoir des modes de description et des profils sémantiques des personnages et de l'environnement représentés, et souvent de manière fortement stylisée et stéréotypée; des configurations de personnages mises en place,

15 Traduction du texte d'une annonce publicitaire en langue allemande du "Robinson Club Baobab. Diani Beach, Kenya ", parue dans différents magazines allemands en 1995.

16 Voir en particulier pour ce qui suit : Roland Barthes, L'aventure sémiologique, Paris, Seuil, 1985 (ici e.a. "Eléments de sémiologie", p. 17-84 et "Sémantique de l'objet ", p. 249-260); Philippe Hamon, Introduction au discours descriptif, Paris, Hachette, 1981; et Erika Fischer-Lichte, "Inszenierung des Fremden. Zur (De-) Konstruktion semiotischer Systeme", Erika Fischer-Lichte (éd.), TheaterAvantgarde. Wabrnebmung - Köpper - Spracbe. Tübingen/Basel, Francke, 1995 (Coll. UTB n ${ }^{\circ} 1807$ ), p. 156-241. 
selon une logique généralement ethnique et raciale, dans le cas des formes de représentations exotiques; ainsi que des types de récits imaginés, découlant souvent des contraintes propres à des genres littéraires donnés (récit d'aventure, récit de découverte, récit utopique, etc.).

Un ouvrage comme Le roman d'un spabi de Pierre Loti donne à lire une étroite interrelation entre ces trois niveaux d'analyse sémiologique. À l'issue tragique des personnages principaux - le soldat colonial français ("spahi") Jean Peyral, son amante sénégalaise Fatou-gaye et leur fils - qui meurent d'une mort violente à la fin, correspondent des registres sémantiques constitutifs à la fois des personnages et des paysages africains dans le roman. Soutenus en même temps par un pouvoir de fascination délibérement mis en récit, des composantes majeures traversent le texte tels des leitmotive, comme "le soleil terne et rouge", "l'intense lumière tropicale", "l'air lourd", "le sable infini " et "excès de chaleur et de lumière". L'Afrique, "merveilleux pays de chasse, de vertu et de forêts " ${ }^{17}$, apparaît en même temps, à d'autres endroits du texte et de manière de plus en plus intense, anticipant ainsi la fin tragique du récit, comme un "grand brasier de feu blanc", pourvu d'un "ciel d'un gris violacé, d'un gris d'étain, qui était tout terni par des miasmes de marais " 18.

Le profil sémantique des Africains représentés, caractérisé par une fascination certaine pour une esthétique et une sensualité différentes ("une grâce de nègre, un charme sensuel, une puissance de séduction matérielle, quelque chose d'indéfinissable " ${ }^{19}$, comme le souligne le narrateur), est dominé par des modes de perception raciaux. Fatou-gaye, l'amante du soldat français Jean Peyral, paraît ainsi caractérisée, dans le roman de Loti, par son type ethnique pur:

Le type khassonké dans toute sa pureté: une fine petite figure grecque, avec une peau lisse et noire comme de l'onyx poli, des dents d'une blancheur éclatante, une extrême moblité des yeux, deux larges prunelles de jais sans cesse en mouvement, roulant de droite et de gauche sur un fond d'une blancheur bleuâtre, entre deux paupières noires. ${ }^{20}$

17 Loti, Roman d'un spabi, e.a. p. 181, p. 125.

18 Ibid., p. 125.

19 Ibid., p. 100

20 Ibid., p. 33-34. p. 63. 
60

Représentée également, à travers l'optique de Peyral, comme un "être inférieur", Fatou-gaye est caractérisêe dans d'autres passages du texte comme "dissimulée et menteuse", marquée par le "malice" et la "perversité", adorant les fétiches, consultant des marabouts, à qui toute une sémantique métaphorique de l'animalité donne une profonde ambivalence: "pattes de singe [...], petites, délicates", ayant "quelque chose de pas humain qui était effrayant", "toujours propre comme une petite chatte habillee de blanc" 21 . Et, enfin, elle apparaît comme "fruit savoureux du Soudan, mûri hâtivement par le printemps tropical, gonflé de sucs toxiques, rempli de voluptés malsaines, enfiévrées, inconnues..... 22

L'analyse du profil sémantique de Fatou-gaye, à la fois pléthorique et profondément ambivalent, renvoie au niveau de l'analyse sociocritique qui cherche à ancrer le texte dans les courants idéologiques d'une époque. Loti représente, en effet, le discours dominant de l'idéologie coloniale française de la phase de la Troisième République (1871-1914), qui visait à la soumission essentiellement militaire et administrative des territoires conquis, mais s'opposait en même temps à toute politique d'intégration culturelle et éducative, ainsi qu'au mélange des populations africaines et françaises ${ }^{23}$. Le message idéologique, ancré doublement dans la logique du récit et dans les différents registres descriptifs, est à cet égard sans ambiguïté.

La mise en perspective interdiscursive fait ressortir, enfin, des clivages à la fois diachroniques et synchroniques, à l'égard du paradigme de représentation de l'exotisme. Une même figuration de signes - par exemple le personnage de l'Africain représenté dans un paysage de palmiers et de forêt tropicale - peut ainsi renvoyer à des formations discursives et des types d'énoncés très divers. Chez Bernardin de Saint-Pierre, il renvoie, dans le roman Paul et Virginie (1788), à la critique de l'esclavage des nègres et à l'utopie d'une société égalitaire rêvée par l'auteur. Un siècle plus

21 Ibid., p. 91-92, 76-77.

22 Ibid., p. 63.

23 Voir sur ce sujet Raoul Girardet, L'idée coloniale en France de 1871 à 1962, Paris, La Table Ronde, coll. "Pluriel ", $\mathrm{n}^{\circ}$ 8338, 1962, p. 21-171; et HansJürgen Lüsebrink, Scbrift, Buch und Lektüre in der französiscbsprachigen Literatur Afrikas. Zur Wabrnebmung und Funktion von Scbrifticbkeit und Bucblektüre in einem kulturellen Epocbenumbrucb der Neuzeit, Tübingen, Niemeyer, coll. "Mimesis", t. 5, 1990, p. 24-33. 
tard et dans la période d'expansion impérialiste de la fin du $\mathrm{XIX}^{\mathrm{e}}$ siècle, il impliquera chez Loti, dans Le roman d'un spabi, une vision anthropologique très ambivalente, basée sur l'imaginaire d'une nécessaire et originelle pureté des races: vision proche des conceptions raciales d'un Gobineau, par exemple, mais opposée en même temps à des discours coloniaux concurrents, fondés sur une anthropologie émancipatrice dans le sillage des Lumières, telle qu'elle est représentée par exemple par l'écrivain et administrateur Georges Hardy ${ }^{24}$. Une image stéréotypée, mais pourvue d'un impact social considérable - comme l'affiche Banania (1917) montrant un tirailleur sénégalais au large sourire devant une caisse de cacao de la marque "Banania" et incarnant pour plusieurs générations de Français la figure même de l'Africain -, renvoie pour sa part beaucoup plus au discours politique et pédagogique, représenté par G. Hardy et axé autour de la conception du paternalisme et de ses soubassements anthropologiques (l'image des Noirs comme de "grands enfants"; vision de leur capacité d'évolution dirigée), qu'à l'imaginaire profondément ethnique et racial présent dans l'œuvre de Pierre Loti.

L'analyse de la perception littéraire de l'Autre, loin de constituer uniquement un "thème" ou une "image ", dépasse ainsi largement, notamment à travers les niveaux d'analyse sociocritique et discursif, le domaine de la littérature proprement dite. Dans cette perspective, une critique littéraire interculturelle implique forcément une dimension constructiviste qui constitue son objet à travers une série de concepts et de questionnements définis. Elle place un texte littéraire, étudié dans une première étape à travers ses registres sémantiques et sa logique narrative, dans un réseau variable de formations idéologiques et discursives représentant, en l'occurrence, des figures d'altérité.

\section{III - Clivages de la postmodernité}

L'évolution littéraire et culturelle de la postmodernité - liée à la fois à la disparition des avant-gardes modernes depuis la fin

24 Voir sur ce sujet Hans-Jürgen Lüsebrink, "Acculturation coloniale et pédagogie interculturelle - L'œeuvre de Georges Hardy", Papa Samba Diop (êd.), Sénégal-Forum. Littérature et bistoire. Werner Glinga in memoriam (1945-1990), Frankfurt/M., IKO - Verlag für Interkulturelle Kommunikation, 1995 ( Studien zu den frankophonen Literaturen außerhalb Europas*, t. 16), p. 113-122. 
62

des années cinquante et l'avènement massif des mass-médias électroniques (télévision, vidéo) au cours des années soixante a déplacé certaines structures de la perception de l'Autre établies au $\mathrm{XvI}^{\mathrm{e}}$ siècle et, en même temps, a formulé de nouveaux défis à la réflexion méthodologique y relative. On peut, en effet, constater trois tendances d'évolution radicalement divergentes.

D'une part, on relève une "mise en relation généralisée" (Patrick Chamoiseau) des sociétés et cultures du globe, à travers de nouveaux phénomènes de masse à échelle planétaire, depuis la fin des années cinquante, comme le tourisme de masse, la mondialisation des industries cinématographiques et la diffusion massive de la télévision dans l'ensemble des sociétés et à travers toutes les couches sociales. Ces phénomènes ont non seulement intensifié les flux d'échanges d'informations et d'images - également, et surtout, d'images de l'Autre - ainsi que les phénomènes de traduction et d'appropriation d'éléments provenant d'autres cultures, mais ont également renforcé des processus de stéréotypisation. La publicité touristique comme noyau discursif des représentations d'autres cultures dans les sociétés contemporaines, pourvue d'une complexité extrêmement réduite, en est un exemple particulièrement caractéristique. Les paratextes (illustrations, pages de couverture, préfaces, etc.) souvent brefs, suivant une logique de marketing culturel et de promotion commerciale et accompagnant les traductions de livres à l'étranger, donnent à lire le même phénomène, dans un domaine plus particulièrement littéraire 25 .

D'autre part, la postmodernité a généré, sur le plan littéraire, mais aussi dans des médias comme le film, la musique et la peinture, de nouvelles formes d'écriture, et de textualité en général, qui mettent en cause les distinctions dichotomiques héritées du $\mathrm{XIX}^{\mathrm{e}}$ et de la première moitié du $\mathrm{xx}^{\mathrm{e}}$ siècle, constitutives notamment des discours nationalistes et coloniaux. Cette mise en cause du clivage entre identité de Soi et perception de l'Autre est d'abord ancrée dans un renversement du regard et une prise de

25 Voir sur cette problématique de la perception de l'Autre dans des formes paratextuelles liées aux traductions Alben Gouaffo, "Fremdwahrnehmung im Paratext ", Bernhard Lenz/Hans-Jürgen Lủsebrink (éds.), Fremabeitserfabrung und Fremdbeitsdarstellung in okzidentalen Kulturen. Tbeorieansätze, Medien/Textsorten, Diskursformen, Passau, Roth, 1996 (Coll. PINK t. 4) (sous presse). A. Gouaffo (Saarbrücken) prépare sur ce sujet, à partir d'un corpus de traductions de romans africains en allemand, une thèse de doctorat (à paraître en 1998). 
parole de ceux qui étaient, jusque dans les années cinquante du $\mathrm{xx}^{\mathrm{e}}$ siècle, essentiellement des objets de discours, à travers les médias de l'écriture (littérature, presse, discours scientifique), l'image imprimée (photographies ${ }^{26}$, illustrations de livres, affiches) et du film. Le titre programmatique du livre de B. Ashcroft et de ses co-auteurs, The Empire writes back (1989) ${ }^{27}$, renvoie, de manière manifestaire, à ce déplacement radical des modes de perception des sociétés et cultures non occidentales. Même si le renversement du regard et la constitution d'une contre-image restèrent, surtout dans une première phase d'évolution marquée dans les cultures francophones africaines et caraïbes par le mouvement de la Négritude, profondément imprégnées par une pensée dichotomique et raciale qui consista à inverser les rôles et les significations données par la pensée coloniale aux événements historiques et aux phénomènes culturels, il déboucha sur de multiples formes d'"ethnologie de l'intérieur" ayant souvent recours au média de la littérature comme forme d'expression. Des écrivains comme Ousmane Sembène au Sénégal, Ngugi wa Thiong'o au Kenya et Nurrudin Farah en Somalie représentent, en effet, des visions littéraires de l'intérieur de leurs sociétés et cultures, qui ont mis en cause non seulement les discours coloniaux, mais également les discours occidentaux (les "hétéro-images", selon la terminologie de l'imagologie comparatiste) sur elles. L'exploration littéraire du monde qui avait accompagné l'expansion des pays européens outre-mer depuis le $\mathrm{xvI}^{\mathrm{e}}$ siècle et généré des paradigmes de perception comme l'exotisme, a ainsi cédé le pas, depuis les années 1930, à un changement de perspective au sein duquel les œuvres pionnières de Michel Leiris et de Pierre Segalen ont servi de relais et de catalyseurs à la prise de parole des Autres et leur "des-exotisation" littéraire et culturelle ${ }^{28}$.

26 Voir sur ce sujet l'excellent livre de Petra Bopp, Fern-Geseben. Französiscbe Bildexpeditionen in den Orient, 1865-1893, Marburg, Jonas, 1995. Je remercie Ulrike Wanitzek (Université de Bayreuth, Allemagne) d'avoir attiré mon attention sur cet ouvrage.

27 Bill Ashcroft/Gareth Griffiths/Hellen Tiffin, The Empire writes back. Theory and practice in post-colonial literatures, London/New York, Routledge, 1989, 5- éd. 1994.

28 Voir en particulier la réédition des ouvrages de Michel Leiris, Miroir de l'Afrique. Édition établie, présentée et annotée par Jean Jamin, avec la collaboration de Jacques Mercier pour les textes ayant trait à l'Ethiopie, Paris, Gallimard, 1966 (comportant e.a. L'Afrique Fantôme, 1934); Victor Segalen, Essai sur l'exotisme, une estbétique du divers (1901), rééd. Paris, Fata Morgana/Le 
64

La troisième tendance constitutive de modes de perception nouveaux de la postmodernité sont les "écritures métisses" qui mettent en cause les conception identitaires propres à l'imaginaire ethnique et racial des $\mathrm{xxx}^{\mathrm{e}}$ et $\mathrm{xx}^{\mathrm{e}}$ siècles. Des écrivains comme Derek Walcott de Trinidad, le Malien Yambo Ouologuem, les Ivoiriens Jean-Marie Adiaffi et Amadou Kourouma, le Nigérien Wole Soyinka et l'auteur indien Vikram Seth contestent toute délimitation nette entre soi-même et l'Autre, entre les cultures occidentales et les cultures non-européennes, ces dernières étant restées fortement marquées et imprégnées, à travers de multiples formes de syncrétisme, par l'expansion coloniale outre-mer. Dans des ceuvres-phares comme Omeros (1990) de Derek Walcott, Texaco (1992) de Patrick Chamoiseau, Le quatrième siècle (1964) d'Edouard Glissant ou Monnè, outrages et défis (1990) d'Amadou Kourouma se dessine l'objectif d'ancrer cette mise en relation gênéralisée dans une esthétique littéraire complexe et pluriculturelle, et de créer ainsi un "imaginaire de la diversité " (P. Chamoiseau), fondé non pas sur l'affirmation d'éthnicités "pures", mais sur des formes de métissage à composantes extrêmement variables. L'Autre est ici perçu, à travers différentes composantes du texte (personnages, environnements, logique narrative, interventions du narrateur, intertextualité) comme un élément constitutif de la propre identité issue d'un processus de métissage ${ }^{29}$. "Crai-

Livre de Poche, coll. "Biblio Essais", n 4042, 1986; et Victor Segalen, Les Immémoriaux (1907), rééd. Paris, Seuil, coll. "Points", no R111, 1979. Voir sur cette problématique aussi le chap. "Du monde exotique au Tiers Monde" du livre de Jean-Marc Moura, Lire l'Exotisme, Paris, Dunod, 1992, p. 173-188; et János Riesz, "Exotismus" als Kampfbegriff. Zum Streit um die "richtige" Kolonialliteratur in Frankreich (1870-1939)", Kulturrevolution. Zeitscbrift für angewandte Diskurstbeorie, $\mathrm{n}^{\circ} 32-33,1995$, p. $75-87$, en particulier p. 83.

29 Voir sur cette problématique e.a. Amadou Koné, "Bilinguisme et écriture du français : écrire deux langues à la fois ", Le Français aujourd'bui, une langue à comprendre. Mélanges offerts à Jürgen Olbert. Éd. par Gilles Dorion, FranzJoseph Meißner et Ulf Wielandt, Frankfurt/M., Diesterweg, 1992, p. 397-409; János Riesz, "Afrikanische Literatur im Zeitalter der Postmoderne", János Riesz, Koloniale Mythen - afrikanische Antworten. Europaisch-afrikanische Literaturbeziebungen I, Frankfurt/M., Verlag für Interkulturelle Kommunikation, 1993 (Studien zu den frankophonen Literaturen außerhalb Europas t. 1), p. 3-24; Ute Fendler, Interkulturalität in der frankopbonen Literatur der Karibik. Der europaisch-afrikaniscb-amerikanische Intertext im Romanwerk ton Maryse Condé, Frankfurt/M., Verlag für Interkulturelle Kommunikation, 1994 ("Studien zu den frankophonen Literaturen außerhalb Europas ", t. 4); et le numéro thématique de la revue allemande Neue Rundschau, $\mathrm{n}^{\circ} 107$, 1995, p. 1-84, sur "Der postkoloniale Blick - eine neue Weltliteratur?", 
gnant cet inconfortable magma", écrivent à ce sujet J. Bernabé, P. Chamoiseau et $\mathrm{R}$. Confiant dans Éloge de la créolité (1989), "nous avons vainement tenté de le figer dans des ailleurs mythiques (regard extérieur, Afrique, Europe, aujourd'hui encore, Inde ou Amérique), de chercher refuge dans la normalité close des cultures millénaires, sans savoir que nous étions l'anticipation du contact des cultures, du monde futur qui s'annonce déjà " 30 .

Il peut à première vue sembler paradoxal que ces trois tendances, apparemment contradictoires et très divergentes, qui caractérisent la post-modernité littéraire et culturelle, à savoir la globalisation des formes de culture de masse, et des images stéréotypées de l'Autre qu'elles véhiculent, d'une part, la "prise de parole" "ethnique" des Autres, et enfin la genèse d'une culture métissée et pluriculturelle, provoquent des défis méthodologiques partiellement semblables. Elles incitent, en effet, à focaliser l'attention, sur le plan de l'analyse idéologique et interdiscursive, sur les phénomènes de réception et de transfert d'une aire culturelle et linguistique à une autre, sous ses multiples formes (adaptation, réécriture, transposition, traduction, réception productive, syncrétisme culturel ${ }^{31}$ ). La perception de l'Autre en littérature, que ce soit au niveau des personnages et de l'environnement représentés, ou à travers une représentation d'ensemble donnée d'une autre culture dans une ouvre littéraire, ne saurait donc plus être traitée ni comme une "image" ni comme un "thème", comme le présupposait l'“imagologie" comparatiste et l'analyse thématique. L'intensification des échanges entre différents types de discours culturels (littérature, film, presse, peinture), d'une part, et différentes aires culturelles nationales, de l'autre, renvoie à la nécessité de focaliser l'analyse sur les phénomènes interdiscursifs et intermédiatiques - comme "l'exotisme", la "Francophilie ", l' Indianité", "l'Américanophilie" - qui sont de puissants générateurs d'auto- et d'hétéro-images traversant un vaste ensemble de formations discursives (dont la littérature écrite et imprimée).

en particulier les contributions de Klaus Martens (sur D. Walcotc) et Pico Iyer (sur "The Empire writes back").

30 Jean Bernabé, Patrick Chamoiseau et Raphaël Constant, Éloge de la créolité, Paris, Gallimard/ Presses Universitaires Créoles, 1989, p. 26-27.

31 Voir sur ce sujet Hans-Jürgen Lüsebrink, "De l'analyse de la réception littéraire à l'étude des transferts culturels", Discours social Social Discourse, vol. VII, $\mathrm{n}^{\text {os }}$ 3-4, été-automne 1995, p. 39-46. 
66

Mais cette accélération des échanges et mises en relation interculturels incite également à prêter plus d'attention aux similitudes et aux différences, aux formes d'appropriation et d'usage de l'Autre dans des littératures et cultures différentes. L'image de l'Amérique et des Américains, par exemple, façonnée par une culture médiatique et matérielle largement identique et diffusée sur la surface du globe entier, est traversée par des formes d'appropriation et des clivages extrêmement divergents. Malgré des formes de représentation largement comparables, voire identiques, au niveau du profil sémantique des personnages et des registres de description de l'environnement urbain des grandes villes américaines, des œuvres comme Volkswagen Blues (1983) de l'écrivain québecois Jacques Poulin et Patron de New York (1964) de l'auteur ivoirien Bernard Binlin Dadié se distinguent par des significations et des fonctions radicalement différentes, quant à la perception qu'elles donnent à lire de la culture USaméricaine qu'ils visitent et qu'ils retracent tous deux dans des romans à caractère autobiographique. Vouloir saisir ces deux images de l'Autre, à la fois largement comparables, et même identiques sur certains plans, et foncièrement différentes, signifie d'abord, dans la perspective d'une critique littéraire interculturelle, de transgresser l'autonomie souvent revendiquée de la littérature et des discours critiques qui s'y greffent; mais aussi d'abandonner l'illusion des possibilités d'existence d'une autonomie des champs littéraires nationaux à partir desquels l'on serait en mesure de distinguer, avec netteté et précision méthodologique, des identités fictionnelles comme l'image de Soi et l'image de l'Autre. 